\title{
Abiraterone acetate for early stage metastatic prostate cancer: patient selection and special considerations
}

This article was published in the following Dove Press journal:

Therapeutics and Clinical Risk Management

\author{
Pietro Castellan' \\ Michele Marchioni² \\ Roberto Castellucci' \\ Piergustavo De Francesco ${ }^{2}$ \\ Romina lantorno' \\ Luigi Schips ${ }^{1,2}$ \\ Luca Cindolo' \\ 'Department of Urology, \\ ASL02 Abruzzo, Chieti, Italy; \\ 2Department of Urology, University \\ "G. d'Annunzio", Chieti, Italy
}

Correspondence: Pietro Castellan Department of Urology, ASL02 Abruzzo,

Via dei vestini, 66100 Chieti, Italy

Tel +39340810 0333

Fax +39087 I 358253

Email castellanpietro@gmail.com

\begin{abstract}
In recent years, therapeutic advances, together with new medication sequences and combinations, have improved outcomes for prostate cancer. For a long time, androgen deprivation therapy (ADT) has been the standard of care for newly diagnosed, metastatic prostate cancer, first as a standalone therapy and then in combination with taxane-based regimens. More recently, the addition of abiraterone acetate to ADT to achieve complete androgen blockade has proven beneficial for the treatment of metastatic hormone-resistant prostate cancer and metastatic hormone-sensitive prostate cancer (mHSPC). In this review, we summarize recent findings on the early use of abiraterone in mHSPC and discuss survival benefits as reported in clinical trials. On the basis of existing data, abiraterone in combination with ADT could be considered a new standard of care for patients affected by mHSPC.
\end{abstract}

Keywords: prostate cancer, metastatic hormone-sensitive prostate cancer, androgen receptor, hormonal therapy, abiraterone acetate, prostate castration naïve

\section{Introduction}

Prostate cancer is the second leading cause of cancer deaths in men worldwide, ${ }^{1}$ accounting for $8 \%$ of all deaths from cancer according to a recent estimate (estimated deaths in 2017, $n=26,730){ }^{2}$ Over the last decade, however, the average survival rate for metastatic prostate cancer ( $\mathrm{mPC}$ ) has improved with the introduction of new pharmacological therapies. ${ }^{3}$ Until 2015, the treatment of $\mathrm{mPC}$ had remained essentially unchanged since Huggins first published his study on the effectiveness of androgen-deprivation therapy (ADT) in $1941 .{ }^{4}$ While the majority of patients show an initial response to ADT, some will develop resistance and progress toward castration-resistant prostate cancer (CRPC) after 18-36 months from the initial biochemical response. ${ }^{5}$ A growing number of therapeutic options are now available for metastatic castration-resistant prostate cancer (mCRPC). One of these is abiraterone acetate (AA), the efficacy of which, in this setting has triggered a number of evaluation studies in the metastatic hormone-sensitive prostate cancer (mHSPC) population. Two pivotal randomized controlled trials (RCTs) explored the efficacy and safety of AA in mHSPC patients, namely, the LATITUDE study (A Randomized, Double-blind, Comparative Study of Abiraterone Acetate Plus Low-Dose Prednisone Plus ADT Versus ADT Alone in Newly Diagnosed Subjects With High-Risk, Metastatic Hormone-naive Prostate Cancer [mHNPC] $)^{6}$ and the $\mathrm{G}$ arm of the STAMPEDE study (Systemic Therapy in Advancing or Metastatic Prostate Cancer: Evaluation of Drug Efficacy). ${ }^{7}$ Both compared standard ADT and ADT plus concurrent AA for men with mHSPC, showing prolonged survival and better clinical outcomes. 
In this review, we discuss the possible role of AA in the treatment of mHSPC.

\section{AA}

AA is an irreversible inhibitor of CYP17A1 (also known as 17, 20 lyase and 17- $\alpha$ hydroxylase), a member of the CYP/CYP450 family that converts pregnanes into steroid hormones, including androgen precursors. ${ }^{8} \mathrm{AA}$ is able to block androgen production in the testis, the adrenal gland, and in prostate tumors, thus preventing tumor growth. Its main side effects are those related to increased mineralocorticoid levels, such as hypertension and hypokalemia. Higher mineralocorticoid concentrations are consequent to CYP17A1 inhibition and increased substrate availability for the mineralocorticoid production cascade. Aldosterone levels are normalized by treatment with either prednisone or prednisolone on account of the negative feedback of those molecules on steroid production. A 1,000 mg/daily plus prednisone or prednisolone $10 \mathrm{mg} /$ daily was approved by the US Food and Drug Administration (FDA) in 2011, after a number of trials showed survival and quality of life (QoL) benefits in mCRPC patients following docetaxel therapy. ${ }^{9}$ In 2014, AA was also approved for chemo-naïve mCRPC patients. ${ }^{10}$

\section{Abiraterone in the setting of castration- sensitive $\mathrm{MPC}$}

Given the evidence in favor of AA in mCRPC, its effectiveness in combination with ADT was evaluated also in patients with locally advanced prostate cancer and $\mathrm{mHSPC}^{11,12}$

\section{Evidence from clinical trials}

The effect of AA with prednisone in addition to ADT on mHSPC survival was tested in two large RCTs. Specifically, the use of AA in this setting was studied in the STAMPEDE arm $\mathrm{G}$ and LATITUDE clinical trials.

STAMPEDE was a multi-arm, multi-stage trial designed to assess whether ADT in combination with other treatments may be associated with better survival outcomes than ADT alone in the first-line setting. ${ }^{7}$ Between November 2011 and January 2014, a total of 1,917 patients were randomized to ADT plus AA with prednisone or to ADT alone. Of these patients, $52 \%$ had metastatic disease, $20 \%$ node-positive or node-indeterminate non-metastatic disease, and 28\% nodenegative non-metastatic disease. Overall, 95\% had newly diagnosed prostate cancer. The primary outcome was overall survival, and the intermediate primary outcome was failurefree survival (defined as radiological, clinical, or biochemical progression or death from prostate cancer). In the interim analysis, the restricted mean failure-free survival time was 43.9 months in the combination group and 30.0 months in the ADT alone group. The combination group also had better overall survival than the ADT alone group (HR for death, 0.63 ; 95\% CI, 0.52-0.76, $P<0.001)$. A failure-free survival advantage for the combination group was apparent both in the metastatic (HR, 0.31, CI, 0.26-0.37) and in the nonmetastatic (HR, 0.21, CI, 0.15-0.31) groups. Conversely, an overall survival advantage was shown in metastatic patients (HR, 0.61, CI, 0.49-0.75) but not in the non-metastatic group (HR, 0.75, CI, 0.48-1). ${ }^{7}$ These findings should be interpreted with caution because follow-up in non-metastatic patients may have been immature and the number of events not sufficient to reach robust conclusions on overall survival. ${ }^{13}$

The LATITUDE trial enrolled 1,199 patients with newly diagnosed, high-risk, metastatic, castration-sensitive prostate cancer (defined as high-risk by any two of three criteria: Gleason score of 8-10, at least three bone lesions, and a measurable visceral disease), all randomly assigned to receive either ADT plus AA with prednisone or ADT with placebo. The primary endpoints were overall survival and radiographic progression-free survival. In the interim analysis (median follow-up, 30.4 months), both endpoints favored the AA arm over the placebo group (the HRs for death and for disease progression or death were 0.62 and 0.39 , respectively; all $P<0.001)$. Better outcomes were also observed for all secondary endpoints, namely time until pain progression, time to subsequent therapies, initiation of chemotherapy, PSA progression, and symptomatic skeletal events. These findings prompted the independent data and safety monitoring committee to unblind the study to allow for treatment crossover. ${ }^{6}$

In a recent meta-analysis, Rydzewska et al analyzed the results from these two trials following a systematic review of the literature. ${ }^{14}$ The primary outcome of interest was overall survival, while the secondary outcomes were clinical/ radiological progression-free survival and grade III-IV and grade $\mathrm{V}$ toxicity for each trial. The meta-analysis showed an impressive concordance of results in the two trials, with very similar HRs (Table 1). This consistency was even more striking when considering progression-free survival. Although the definition of progression differed in the STAMPEDE and LATITUDE trials, the results were quite similar (HRs for progression, 0.43 and 0.47 , respectively). There were no differences in the overall survival benefit as measured by the Gleason sum score, performance status, or nodal status; however, the magnitude of benefit was greater in younger men and less pronounced in older men. In addition, 
Table I Comparison of the STAMPEDE and LATITUDE trials

\begin{tabular}{|c|c|c|}
\hline & STAMPEDE (James et al, 20I7) & LATITUDE (Fizazi et al, 20I7) \\
\hline Patient population & $\begin{array}{l}\text { Non-metastatic, node neg: } 28 \% \text {; non-metastatic, node pos: } \\
20 \% \text {; metastatic: } 52 \%\end{array}$ & Metastatic: $100 \%$ \\
\hline Study design & Randomized, Phase III trial with multi-group, multistage platform design & Randomized, Phase III controlled trial \\
\hline Primary endpoints & Overall survival; failure-free survival & Overall survival; r-PFS \\
\hline Study treatment & $\begin{array}{l}\mathrm{ADT}+\text { abiraterone/prednisolone }{ }^{\mathrm{a}}+\text { radiotherapy (in non-metastatic } \\
\text { patients) }\end{array}$ & ADT + abiraterone/prednisone ${ }^{a}$ \\
\hline Control treatment & $A D T+$ radiotherapy (in non-metastatic patients) & $\begin{array}{l}\text { ADT + dual placebos (for abiraterone } \\
\text { and prednisone) }\end{array}$ \\
\hline Treatment duration & $\begin{array}{l}\text { Non-metastatic and radiotherapy: } 2 \text { years (planned); metastatic or } \\
\text { non-metastatic, but not radiotherapy: until progression }\end{array}$ & Until progression \\
\hline Total sample size & I,917 ( 1,002 metastatic patients) & 1,199 \\
\hline HR for death $(95 \% \mathrm{Cl})$ & $0.63(0.52-0.76)$, favoring abiraterone & $0.62(0.5 \mathrm{I}-0.76)$ favoring abiraterone \\
\hline $\begin{array}{l}\text { Other primary } \\
\text { endpoints }\end{array}$ & $r-P F S: H R=0.47$ & $\begin{array}{l}\text { Treatment failure } H R=0.29 \text {, with evidence } \\
\text { of non-proportional hazards }\end{array}$ \\
\hline
\end{tabular}

Note: aPrednisolone and prednisone are standard, co-administered medications used with abiraterone to prevent hyperaldosteronism (negative feedback). Abbreviations: ADT, androgen deprivation therapy; neg, negative; pos, positive; r-PFS, radiographic progression-free survival.

higher rates of acute grade III-IV cardiac, vascular, and hepatic toxicities were shown in the AA group. Interestingly, no excess of deaths from treatment was observed. The authors concluded that adding AA to the standard therapy is clinically effective and offers an alternative to docetaxel for men starting first-line treatment for HSPC.

\section{Abiraterone vs docetaxel in metastatic castration-sensitive prostate cancer}

The LATITUDE and STAMPEDE trials clearly demonstrate how ADT plus AA is superior to ADT alone in the treatment of newly diagnosed mHSPC. In 2015, two other trials showed that adding docetaxel to ADT results in improved survival in men with a recent diagnosis of $\mathrm{mPC} .^{12,15}$ This raises an important question for the insider, namely, how to correlate this finding with data on AA showing a similar advantage over early-stage treatment. The trials that assessed early docetaxel and AA used various patient populations and slightly different definitions of "high-risk" or "high-volume" disease.

In a meta-analysis, Wallis et al analyzed 6,067 patients from the recently published early docetaxel (GETUG-AFU, CHAARTED, and STAMPEDE) and early abiraterone (LATITUDE and STAMPEDE) trials. ${ }^{16}$ The analysis demonstrated no statistically significant overall survival differences for abiraterone-ADT when compared with docetaxel-ADT for patients with high-risk cancer or mHSPC. Moreover, abiraterone-ADT showed better performance in younger patients (HR, 0.77, 95\% CI, 0.60-1.004). While these data may suggest a potential preference for abiraterone-ADT over docetaxel-ADT for initial treatment, both therapies are feasible for newly diagnosed mHSPC. Data from two other similar studies ${ }^{17,18}$ suggest a relatively improved efficacy of abiraterone-ADT compared with docetaxel-ADT, again without significant differences in overall survival. For this reason, treatment choices continue to be driven by physician's preference, convenience, and cost rather than relative efficacy. ${ }^{19-21}$ The safety profile for each regimen is also a matter of debate. It is generally accepted that the acute toxicity of docetaxel is higher than that of abiraterone, but this is reversed when taking into account the possible long-term effects of abiraterone/glucocorticoid combined with ADT. ${ }^{22}$ The relative costs of early docetaxel or abiraterone will also need to be considered in increasingly financially burdened health care systems worldwide. ${ }^{21}$

\section{Evidence from guidelines}

All the main guidelines address one question, namely, whether there is an overall survival advantage associated with the addition of AA to ADT in men with mHSPC. The European Association of Urology (EAU) guidelines approve AA in combination with ADT for MCRPC and mHSPC. In 2011, in light of results from a Phase III trial (COU-AA-301) that reported a significant increase in overall survival and radiographic progression-free survival, AA plus prednisone (AAP) was approved as a secondline treatment after docetaxel. ${ }^{23}$ In 2013, after the results of another Phase III trial (COU-AA-302) were published, AAP was adopted as a first-line treatment for mCRPC. ${ }^{24}$ The proven effectiveness of AA in advanced-stage disease prompted investigation into its efficacy also in earlier settings. 
The previously mentioned STAMPEDE and LATITUDE trials showed significant overall survival benefits of up to $38 \%$ at 3 years, with the secondary endpoints favoring the ADT plus AA combination. The EAU guidelines recommend AA for patients with mCRPC before or after docetaxel chemotherapy; castration combined with AAP for all patients whose first presentation is M1 disease and who are fit enough for this regimen; castration alone, with or without an antiandrogen, for patients unfit for castration combined with docetaxel or AAP, or who are unwilling to consider it. ${ }^{25}$

The American Urological Association guidelines consider different scenarios: ${ }^{26} 1$ ) patients with asymptomatic, non-metastatic CRPC may be offered AAP; 2) patients with asymptomatic or minimally symptomatic $\mathrm{mCRPC}$ who are chemo-naïve may receive AAP, enzalutamide, or docetaxel chemotherapy and sipuleucel-T immunotherapy; 3 ) patients with symptomatic $\mathrm{mCRPC}$ and good performance status who are chemo-naïve may receive AAP, enzalutamide, or docetaxel; 4) patients with symptomatic mCRPC, poor performance status, and no prior docetaxel chemotherapy may receive AAP or enzalutamide; 5) patients with symptomatic mCRPC, good performance status, and prior docetaxel chemotherapy may receive AAP, cabazitaxel, or enzalutamide; and 6) patients with symptomatic mCRPC, poor performance status, and prior docetaxel chemotherapy should not be offered AAP.

In February 2008, the National Comprehensive Cancer Network(NCCN)Guidelines(ProstateCancer-Version4.2018) incorporated the FDA's recommendations for approval of AA in combination with prednisone for castration-naïve, $\mathrm{mPC}$. The term "castration-naïve" refers to patients who are not on ADT at the time of progression, including those who have had neoadjuvant, concurrent, or adjuvant ADT as part of radiation therapy provided they have recovered testicular function. The NCCN panel listed AA with $5 \mathrm{mg}$ prednisone once daily as a recommended treatment option with ADT for men with newly diagnosed, castration-naïve, M1 prostate cancer (category 1). However, the available data on survival, failure-free survival (FFS), and follow-up are still insufficient to recommend AA for patients with high-risk or very-highrisk, N0, M0 prostate cancer. ${ }^{27}$

According to the American Society of Clinical Oncology guidelines, ${ }^{28}$ treatment with AA or docetaxel in combination with ADT should be offered to patients with newly diagnosed, non-castrate metastatic disease on account of its proven survival benefit over ADT alone. Specifically, patients with de novo, high-risk, non-castrate metastatic disease (as per the LATITUDE criteria, namely two or more of Gleason score $\geq 8$, more than three bone metastases, and measurable visceral disease) who are fit for treatment with abiraterone should receive ADT and AAP. Patients with low-risk disease may also be offered ADT and AAP (as recommended in the STAMPEDE trial).

At the time of writing, there were insufficient data to recommend which patients should receive abiraterone and which patients should receive docetaxel, and no data to recommend abiraterone plus docetaxel for non-castrate, metastatic disease, either combined or sequentially. The only clear consensus is that the addition of abiraterone to ADT in patients with newly diagnosed $\mathrm{mPC}$ offers a survival benefit over ADT alone. ${ }^{29}$

\section{Adverse events and QoL}

In the LATITUDE trial, the rates of serious adverse events were similar between the two arms. Adverse events resulting in dose modification or interruption were $17 \%$ in the ADT alone arm and $32 \%$ in the AA arm. Treatment discontinuation rates were $10 \%$ in the ADT alone arm and $12 \%$ in the AA arm. Hypertension and hypokalemia were more frequent in the AA group.

In the STAMPEDE trial, the percentage of grade $>3$ adverse events was similar in both arms (ADT alone 11\%, vs AA 15\%). Hypertension, respiratory disorders, and increased AST levels were also associated with AA.

The LATITUDE trial used various tools for population assessment, including the Functional Assessment of Cancer Therapy-Prostate (version 4; FACT-P) protocol, the Brief Fatigue Inventory, and the EurQoL five-dimension five-level (EQ-5D-5L) questionnaire. ${ }^{30} \mathrm{AA}$ had better pain control (37\% risk reduction for worst pain progression; HR, 0.63 ; 95\% CI, 0.52-0.77; $P=0.0001)$ and pain interference progression (33\% risk reduction; HR, 0.67; 95\% CI, 0.56-0.80; $P=0.0001)$. AA also showed improvements in fatigue progression (35\% risk reduction; HR, 0.65 ; 95\% CI, 0.53-0.81; $P=0.0001)$ and fatigue interference progression $(41 \%$ risk reduction; $\mathrm{HR}, 0.59 ; 95 \% \mathrm{CI}, 0.47-0.75 ; P=0.0001)$. AA reduced the risk of health-related QoL deterioration by $15 \%$ (HR, $0.85 ; 95 \% \mathrm{CI}, 0.74-0.99 ; P=0.0322$; HR, $0.85 ; 95 \% \mathrm{CI}, 0.74-0.99 ; P=0.0322$ according to FACT-P). Again, the health status and health utility scores (EQ-5D-5L questionnaire - visual analog scale) showed statistically significant improvements. Reports on QoL data are still pending for the STAMPEDE trial.

\section{Discussion and future perspectives}

Since Huggins demonstrated how the handling of testosterone levels can favorably affect the evolution of prostate cancer, 
ADT has become the mainstay of treatment for mHSPC. ${ }^{31,32}$ After that, only docetaxel, approved in 2004, showed a significant overall survival benefit in patients with $\mathrm{mCRPC}$ (HR, 0.76; overall survival benefit, 2-3 months). ${ }^{33}$ More recently, in 2011, AA was also approved for mCRPC, after demonstrating a further 3.9 months' improvement in median overall survival (HR, 0.65; 95\% CI, 0.54-0.77; $P<0.001$ ) due to its ability to eliminate extragonadal (primarily adrenal) androgen synthesis. Finally, in 2014, the indication for AA was extended as a first-line treatment option also to patients with chemotherapy-naïve $\mathrm{mCRPC}$ on account of a $57 \%$ reduction in the risk of radiographic progressionfree survival (HR, 0.43; 95\% CI, 0.35-0.52; $P<0.0001)^{24}$ coupled with a significant survival benefit (34.7 months; 95\% CI, 32.7-36.8, Pv=0.0033).

Nowadays, efforts are directed toward investigating the efficacy of available drugs in the earlier stages of disease. New combinations are also being studied with the aim to identify the optimal timing and sequence for patients. Treatment success, however, is never a foregone conclusion, given the considerable genotypic and phenotypic differences between ADT-sensitive and ADT-resistant disease. AA was associated with a clear survival benefit in patients at the early stages of disease, probably on account of their better general condition that in turn resulted in a more effective response to treatment. Both the STAMPEDE and LATITUDE trials showed a clear treatment advantage for AA in men with mHSPC. The main difference between the two studies was in the patient population. The STAMPEDE trial investigated a mixed group of patients ( $52 \%$ with metastatic disease and $48 \%$ with non-metastatic disease, plus a subgroup of $28 \%$ with high-risk, localized disease without obvious nodal or metastatic involvement). Also, approximately $40 \%$ of patients with non-metastatic disease were treated with radiation therapy. Conversely, the LATITUDE trial only included metastatic patients, specifically, those with de novo metastatic disease, arbitrarily defined as high risk by any two of three criteria, namely a Gleason score of 8-10, at least three bone lesions, and a measurable visceral disease. Radiotherapy was not used, which prevents a direct comparison of survival rates. Median survival time for the control group in the LATITUDE study was 34.7 months, while the HR for death and the $95 \%$ CI were similar in both trials. Both studies had equally positive results for secondary endpoints (ie, pain, symptomatic skeletal events, and PSA progression).

Understanding differences in the available data may provide the key to identify which patient population stands to benefit the most from AA. ${ }^{34,35}$ Prostate cancer is a heterogeneous disease with a broad risk spectrum and tends to occur predominantly in elderly patients, who already face competing risks from comorbidities. The main focus of the STAMPEDE and LATITUDE trials was on metastatic patients, with compelling results for those with higher volumes of bone disease. For patients with presumed localized prostate cancer, after primary treatment and an often prolonged biochemical response, many develop $\mathrm{mPC}$; in fact, data from the US confirm that approximately only $4 \%$ have de novo metastatic PC. ${ }^{2}$ When biochemical failure appears, existing imaging techniques (bone scan and abdominopelvic computed tomography [CT]) are currently unable to detect metastases in the presence of low PSA levels. The most effective tool in such cases is positron emission tomography/CT (PET/CT) imaging with 18F-Fluciclovine and Ga68-PSMA, which appears to be more sensitive than choline PET/CT in the detection of distant metastases in patients with low PSA levels. ${ }^{36,37}$

Also, although both the STAMPEDE and LATITUDE trials evidenced how early treatment may amplify the therapeutic effects observed in MCRPC, neither study investigated whether administering abiraterone to patients with non-castrate disease is superior to giving chemotherapy to patients with mCRPC, because both had been designed and carried out before the data on docetaxel were reported. In a recent meta-analysis, Wallis et $\mathrm{al}^{16}$ concluded that both approaches are acceptable for patients newly diagnosed with high-risk or hormone-naïve mPC. van Soest and de Wit reached similar conclusions, namely that ADT plus AA or docetaxel prolongs overall survival in patients with mHSPC. ${ }^{38}$ The choice of treatment therefore depends on factors such as physician's preferences, patient's health status, and treatment-associated adverse events. In another recent population-based data analysis, Wallis et al reported that exposure to docetaxel in men being treated for $\mathrm{mCRPC}$ was associated with a significant increase in treatment-related toxicities requiring hospitalization. ${ }^{39}$ However, there seems to be a consensus that treatment with AA may help avoid chemotherapy and its rare but potentially life-threatening complications and may be more suitable in elderly patients or those unfit for chemotherapy. ${ }^{40}$

The STAMPEDE and LATITUDE trials focused on two key aspects, namely the ability to select the correct regimen for the right patient and the need to better identify the mechanisms of drug resistance. In the majority of cases, androgen receptors (ARs) remain the main drivers of resistance: the clinical relevance of AR-Vs in CRPC has been demonstrated and AR-V7 protein is frequently expressed. ${ }^{41}$ 
In a study, Hörnberg et al showed that expression of AR-Vs is increased in CRPC compared to hormone-naïve bone metastases. ${ }^{42}$ In another study, Antonarakis et al reported how $53 \%$ of enzalutamide-resistant patients and $63 \%$ of abiraterone-resistant patients exhibited detectable AR-V7 in circulating tumor cells. ${ }^{43}$ The genomic rearrangement is a key mechanism that promotes AR-Vs' synthesis, ${ }^{44}$ and prostate tumors can escape ADT by maintaining a functional AR through various mechanisms: activation of glucocorticoid receptor, synthesis of androgens in CRPC tissues, AR mutations, AR gene amplification, and AR-Vs. New second-generation AR antagonists with improved efficacy and reduced side effects are under investigation. ${ }^{45}$ Within this framework, several advances have been made, ${ }^{46}$ and the need for additional therapies has been recognized. ${ }^{47}$ In the near future, results from the PEACE-1 trial (A Phase III of ADT+Docetaxel+/-Local RT \pm Abiraterone Acetate in Metastatic Hormone-Naïve Prostate Cancer) ${ }^{48}$ are expected to further clarify the role and optimal timing of AA treatment even in patients in the early stages of disease. ${ }^{14}$ Physicians and researchers need to be wary of the effects of stage migration on early $\mathrm{mPC}$ detection, which in future may be aided by more sensitive techniques, such as PSMA PET/TC imaging, even in the presence of low PSA levels.

\section{Conclusion}

On the basis of existing data, it seems reasonable that AA in combination with ADT should be considered a new standard of care for patients with mHSPC. Findings from the STAMPEDE and LATITUDE trials have already led to new guidelines and recommendations for the management of prostate cancer; however, additional prospective and randomized studies are needed to optimize the sequence and combination of approved drugs.

\section{Disclosure}

The authors report no conflict of interest in this work.

\section{References}

1. Howlader Miller D, Bishop K, et al. Prostate cancer SEER cancer. Stat Rev. 2017:1975-2014.

2. Siegel RL, Miller KD, Jemal A. Cancer Statistics, 2017. CA Cancer J Clin. 2017;67(1):7-30.

3. Berg KD, Thomsen FB, Mikkelsen MK, Ingimarsdóttir IJ, Hansen RB, Kejs AM, Brasso K. Improved survival for patients with de novo metastatic prostate cancer in the last 20 years. Eur J Cancer. 2017;72:20-27.

4. Huggins C. Effect of orchiectomy and irradiation on cancer of the prostate. Ann Surg. 1942;115(6):1192-1200.

5. Wadosky KM, Koochekpour S. Molecular mechanisms underlying resistance to androgen deprivation therapy in prostate cancer. Oncotarget. 2016;7(39):64447-64470.
6. Fizazi K, Tran N, Fein L, et al. Abiraterone plus prednisone in metastatic, castration-sensitive prostate cancer. N Engl J Med. 2017;377(4): $352-360$

7. James ND, de Bono JS, Spears MR, et al; STAMPEDE Investigators. Abiraterone for prostate cancer not previously treated with hormone therapy. N Engl J Med. 2017;377(4):338-351.

8. Scott LJ. Abiraterone acetate: a review in metastatic castration-resistant prostrate cancer. Drugs. 2017;77(14):1565-1576.

9. Fizazi K, Scher HI, Molina A, et al; COU-AA-301 Investigators. Abiraterone acetate for treatment of metastatic castration-resistant prostate cancer: final overall survival analysis of the COU-AA-301 randomised, double-blind, placebo-controlled phase 3 study. Lancet Oncol. 2012;13(10):983-992.

10. Ryan CJ, Smith MR, Fizazi K, et al; COU-AA-302 Investigators. Abiraterone acetate plus prednisone versus placebo plus prednisone in chemotherapy-naive men with metastatic castration-resistant prostate cancer (COU-AA-302): final overall survival analysis of a randomised, double-blind, placebo-controlled phase 3 study. Lancet Oncol. 2015;16(2):152-160.

11. James ND, Spears MR, Sydes MR. Abiraterone in metastatic prostate cancer. $N$ Engl J Med. 2017;377:1696-1697.

12. James ND, Sydes MR, Clarke NW, et al; STAMPEDE investigators. Addition of docetaxel, zoledronic acid, or both to first-line long-term hormone therapy in prostate cancer (STAMPEDE): survival results from an adaptive, multiarm, multistage, platform randomised controlled trial. Lancet. 2016;387(10024):1163-1177.

13. Spears MR, James ND, Sydes MR. 'Thursday's child has far to go'interpreting subgroups and the STAMPEDE trial. Ann Oncol. 2017; 28(10):2327-2330.

14. Rydzewska LHM, Burdett S, Vale CL, et al; STOPCaP Abiraterone Collaborators. Adding abiraterone to androgen deprivation therapy in men with metastatic hormone-sensitive prostate cancer: a systematic review and meta-analysis. Eur J Cancer. 2017;84:88-101.

15. Sweeney CJ, Chen YH, Carducci M, et al. Chemohormonal therapy in metastatic hormone-sensitive prostate cancer. $N$ Engl J Med. 2015; 373(8):737-746.

16. Wallis CJD, Klaassen Z, Bhindi B, et al. Comparison of abiraterone acetate and docetaxel with androgen deprivation therapy in high-risk and metastatic hormone-naïve prostate cancer: a systematic review and network meta-analysis. Eur Urol. 2018;73(6): 834-844.

17. Sydes MR, Spears MR, Mason MD, et al; STAMPEDE Investigators. Adding abiraterone or docetaxel to long-term hormone therapy for prostate cancer: directly randomised data from the STAMPEDE multi-arm, multi-stage platform protocol. Ann Oncol. 2018;29(5):1235-1248.

18. Vale CL, Fisher DJ, Carpenter J, et al. What are the optimal systemic treatments for men with metastatic, hormone-sensitive prostate cancer? A STOPCaP systematic review and network meta-analysis. Ann Oncol. 2017;28(suppl 5):1249-1257.

19. Tan PS, Aguiar P, Haaland B, Lopes G. Addition of abiraterone, docetaxel, bisphosphonate, celecoxib or combinations to androgendeprivation therapy (ADT) for metastatic hormone-sensitive prostate cancer (mHSPC): a network meta-analysis. Prostate Cancer Prostatic Dis. 2018:136.

20. Abdel-Rahman O, Cheung WY. Impact of prior local treatment on the outcomes of metastatic hormone-sensitive prostate cancer: secondary analysis of a randomized controlled trial. Clin Genitourin Cancer. Epub 2018 Jul 21.

21. Xu L, Pachynski RK. Contemporary management of the newly diagnosed prostate cancer patient with metastatic disease at presentation. Curr Urol Rep. 2018;19(10):79.

22. Aggarwal R. Abiraterone or docetaxel plus androgen deprivation in hormone-sensitive prostate cancer: more questions than answers. Eur Urol. 2018;73(6):845-846.

23. de Bono JS, Logothetis CJ, Molina A, et al; COU-AA-301 Investigators. Abiraterone and increased survival in metastatic prostate cancer. N Engl J Med. 2011;364(21):1995-2005. 
24. Ryan CJ, Smith MR, de Bono JS, et al; COU-AA-302 Investigators. Abiraterone in metastatic prostate cancer without previous chemotherapy. N Engl J Med. 2013;368(2):138-148.

25. Mottet NBJ, Briers E. Members of the EAU - ESTRO - ESUR -SIOG Prostate Cancer Guidelines Panel. EAU - ESTRO - ESUR - SIOG Guidelines on Prostate Cancer. Available from: https://uroweb.org/ guideline/prostate-cancer/. Accessed August 2, 2018.

26. American Urological Association. Clinically Localized Prostate Cancer. AUA/ASTRO/SUO Guidelines; 2017. Available from: http://www auanet.org/guidelines/prostate-cancer-clinically-localized. Accessed August 2, 2018.

27. National Comprehensive Cancer Network: (NCCN). Prostate Cancer (Version 4.2018). National Comprehensive Cancer Network; 2018 Available from: http://www.nccn.org/professionals/physician_gls/pdf/ prostate.pdf. Accessed August 2, 2018.

28. Morris MJ, Rumble RB, Basch E, et al. Optimizing anticancer therapy in metastatic non-castrate prostate cancer: American society of clinica oncology clinical practice guideline. J Clin Oncol. 2018;36(15):1521-1539.

29. U.S. Food and Drug Administration. FDA approves abiraterone acetate in combination with prednisone for high-risk metastatic castrationsensitive prostate cancer. FDA; 2018. Available from: www.fda. gov/Drugs/InformationOnDrugs/ApprovedDrugs/ucm596015.htm. Accessed August 2, 2018.

30. Chi KN, Protheroe A, Rodríguez-Antolín A, et al. Patient-reported outcomes following abiraterone acetate plus prednisone added to androgen deprivation therapy in patients with newly diagnosed metastatic castration-naive prostate cancer (LATITUDE): an international, randomised phase 3 trial. Lancet Oncol. 2018;19(2):194-206.

31. Huggins C, Bergenstal DM. Effect of bilateral adrenalectomy on certain human tumors. Proc Natl Acad Sci U S A. 1952;38(1):73-76.

32. Huggins C. Studies on prostatic cancer. Arch Surg. 1941;43(2):209.

33. Tannock IF, de Wit R, Berry WR, et al; TAX 327 Investigators. Docetaxel plus prednisone or mitoxantrone plus prednisone for advanced prostate cancer. $N$ Engl J Med. 2004;351(15):1502-1512.

34. McNamara M, Sweeney C, Antonarakis ES, Armstrong AJ. The evolving landscape of metastatic hormone-sensitive prostate cancer: a critical review of the evidence for adding docetaxel or abiraterone to androgen deprivation. Prostate Cancer Prostatic Dis. 2018;21(3):306-318.

35. Damodaran S, Kyriakopoulos CE, Jarrard DF. Newly diagnosed metastatic prostate cancer: has the paradigm changed? Urol Clin North Am. 2017;44(4):611-621.

36. Malaspina S, de Giorgi U, Kemppainen J, del Sole A, Paganelli G. ${ }^{68} \mathrm{Ga}-\mathrm{PSMA}-\mathrm{PET}$ : added value and future applications in comparison to the current use of choline-PET and mpMRI in the workup of prostate cancer. Radiol Med. Epub 2018 Aug 16.
37. Lengana $\mathrm{T}$, Lawal IO, Boshomane TG, et al. ${ }^{68} \mathrm{Ga}-\mathrm{PSMA}$ PET/CT replacing bone scan in the initial staging of skeletal metastasis in prostate cancer: a fait accompli? Clin Genitourin Cancer. 2018;16(5): $392-401$.

38. van Soest RJ, de Wit R. Irrefutable evidence for the use of docetaxel in newly diagnosed metastatic prostate cancer: results from the STAMPEDE and CHAARTED trials. BMC Med. 2015;13:304.

39. Wallis CJD, Satkunasivam R, Saskin R, Bansal S, Kulkarni GS, Emmenegger U, Nam RK. Population-based analysis of treatment toxicity among men with castration-resistant prostate cancer: a Phase IV study. Urology. 2018;113:138-145.

40. Cindolo L, Natoli C, De Nunzio C, et al. Safety and efficacy of abiraterone acetate in chemotherapy-naive patients with metastatic castration-resistant prostate cancer: an Italian multicenter "real life" study. BMC Cancer. 2017;17(1):753.

41. McGrath MJ, Binge LC, Sriratana A, et al. Regulation of the transcriptional coactivator FHL2 licenses activation of the androgen receptor in castrate-resistant prostate cancer. Cancer Res. 2013;73(16): 5066-5079.

42. Hörnberg E, Ylitalo EB, Crnalic S, et al. Expression of androgen receptor splice variants in prostate cancer bone metastases is associated with castration-resistance and short survival. PLoS One. 2011;6(4): e19059.

43. Antonarakis ES, Lu C, Wang H, et al. AR-V7 and resistance to enzalutamide and abiraterone in prostate cancer. NEngl J Med. 2014;371(11): 1028-1038.

44. Nyquist MD, Dehm SM. Interplay between genomic alterations and androgen receptor signaling during prostate cancer development and progression. Horm Cancer. 2013;4(2):61-69.

45. Coutinho I, Day TK, Tilley WD, Selth LA. Androgen receptor signaling in castration-resistant prostate cancer: a lesson in persistence. Endocr Relat Cancer. 2016;23(12):T179-T197.

46. Gourdin T. Recent advances in the management of metastatic prostate cancer: optimizing use of existing therapies, while searching for novel interventions. Curr Opin Oncol. 2018;30(3):159-164.

47. Komura K, Sweeney CJ, Inamoto T, Ibuki N, Azuma H, Kantoff PW. Current treatment strategies for advanced prostate cancer. Int J Urol. 2018;25(3):220-231.

48. UNICANCER. A Phase III of ADT + Docetaxel +/- Local RT +/Abiraterone Acetate in Metastatic Hormone-Naïve Prostate Cancer (PEACE1). Available from: https://clinicaltrials.gov/ct2/show/ NCT01957436. NLM identifier: NCT01957436. Accessed August 16, 2018.
Therapeutics and Clinical Risk Management

\section{Publish your work in this journal}

Therapeutics and Clinical Risk Management is an international, peerreviewed journal of clinical therapeutics and risk management, focusing on concise rapid reporting of clinical studies in all therapeutic areas outcomes, safety, and programs for the effective, safe, and sustained use of medicines. This journal is indexed on PubMed Central, CAS,

\section{Dovepress}

EMBase, Scopus and the Elsevier Bibliographic databases. The manuscript management system is completely online and includes a very quick and fair peer-review system, which is all easy to use. Visit http://www.dovepress.com/testimonials.php to read real quotes from published authors. 